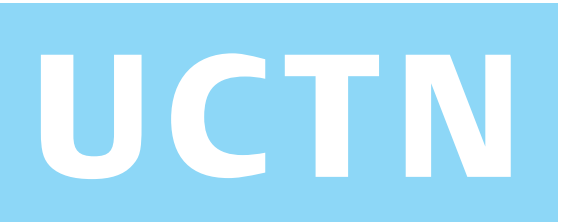

\title{
Gastritis cystica profunda in a patient with no history of gastric surgery
}

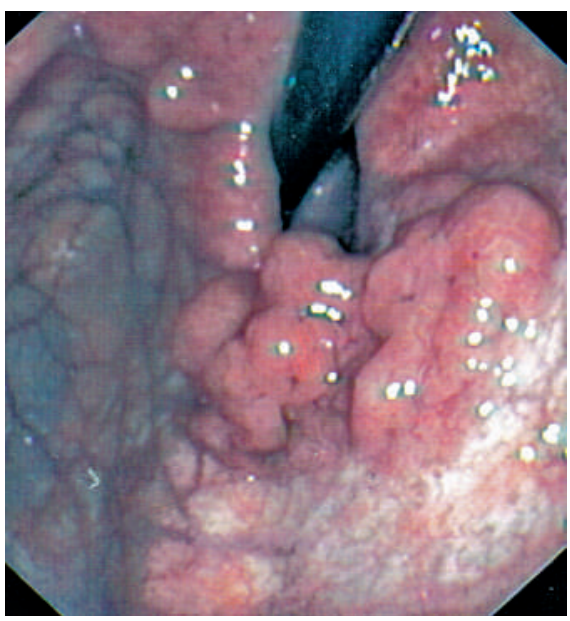

Figure 1 Gastroscopic view showing giant folds along the anterior wall of the fundus.

A 79-year-old woman was admitted because of intermittent epigastric pain. She had no history of gastrectomy or gastroenterostomy. Gastroscopy revealed giant gastric folds along the anterior wall of the fundus (Figure 1). A partial mucosectomy of the lesion showed dilated cystic glands in the basal portion of mucosa, extending to the submucosa through an interruption of the muscularis mucosae (Figure 2). No dysplastic changes were observed. Contrast-enhanced abdominal computed tomography revealed an intraluminal polypoid mass with a thick peripheral wall (Figure 3). Endoscopic ultrasound showed a thickened, echo-poor submucosal layer containing multiple anechogenic areas, indicating the presence of submucosal cysts (Figure 4). These findings were consistent with gastritis cystica profunda. Because of the age of the patient, no surgical resection was performed. A follow-up gastroscopy 3 months after the initial examination showed no macroscopic changes.

In most cases, gastritis cystica profunda develops in patients who have undergone gastroenterostomy, with or without gastric resection, and the lesion usually appears on the gastric side of the anastomosis [1]. It is rarely discovered in an unoperated stomach [2]. The lesions are char-

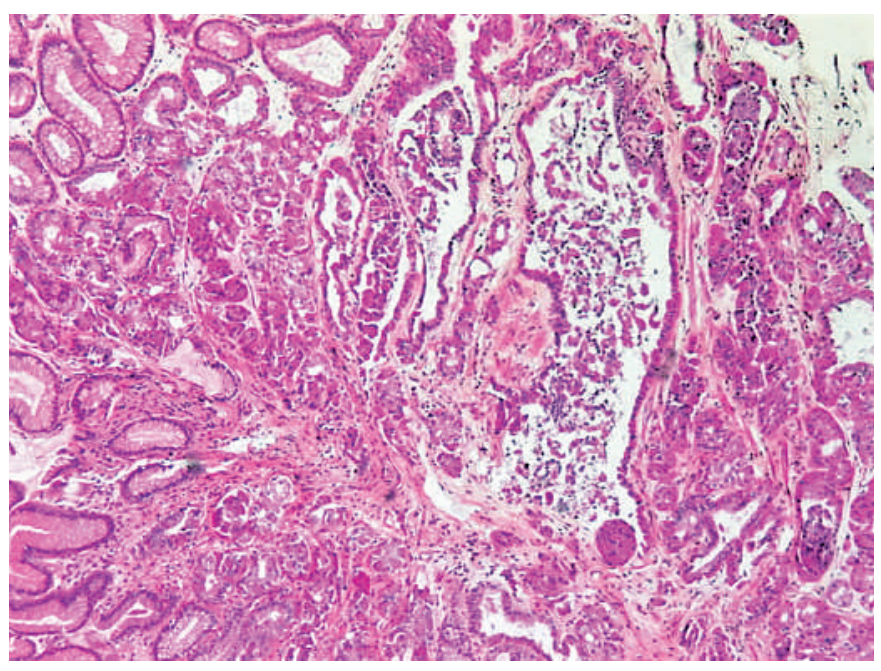

Figure 2 Histological examination of a biopsy specimen showed dilated cystic glands in the basal portion of mucosa.

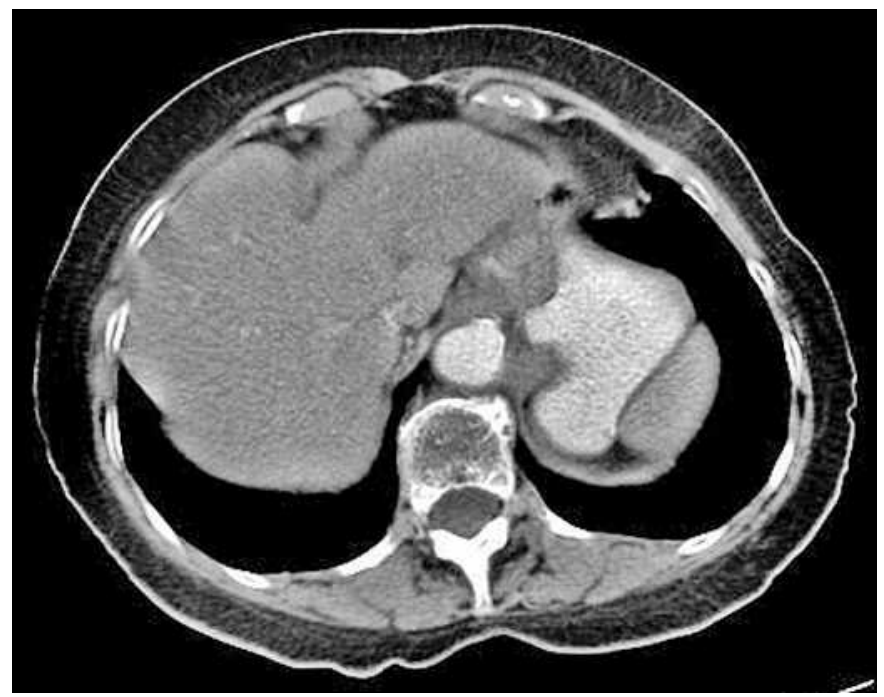

Figure 3 Computed tomographic view showing an intraluminal polypoid mass.

acterized by an interruption of the muscularis mucosae caused by an erosion of the gastric mucosa in chronic gastritis or ischemia: there is elongation of the gastric foveolae and hyperplasia and cystic dilatation of the gastric glands, extending into the submucosal layers [3]. In an operated stomach, the effects of surgery or the presence of suture material could allow epithelial elements to migrate into the submucosa. Gastritis cystica profunda may present as giant gastric folds, as a submucosal tumor, or as an isolated polyp [4]. Endoscopic ultrasound shows an echo-poor, multilocular cystic mass in the thickened submucosal layer. Gastritis cystica profunda is considered to be a potentially precancerous lesion [5], and removal and histopathologic confirmation of the diagnosis is required due to a high reported incidence of carcinoma [4].

Endoscopy_UCTN_Code_CCL_1AB_2AD_3AD

\section{Béchade, J. Desramé, J. P. Algayres}

Department of Gastroenterology, Val de Grâce Hospital, Paris, France.

DOI: $10.1055 / \mathrm{s}-2006-945070$ 


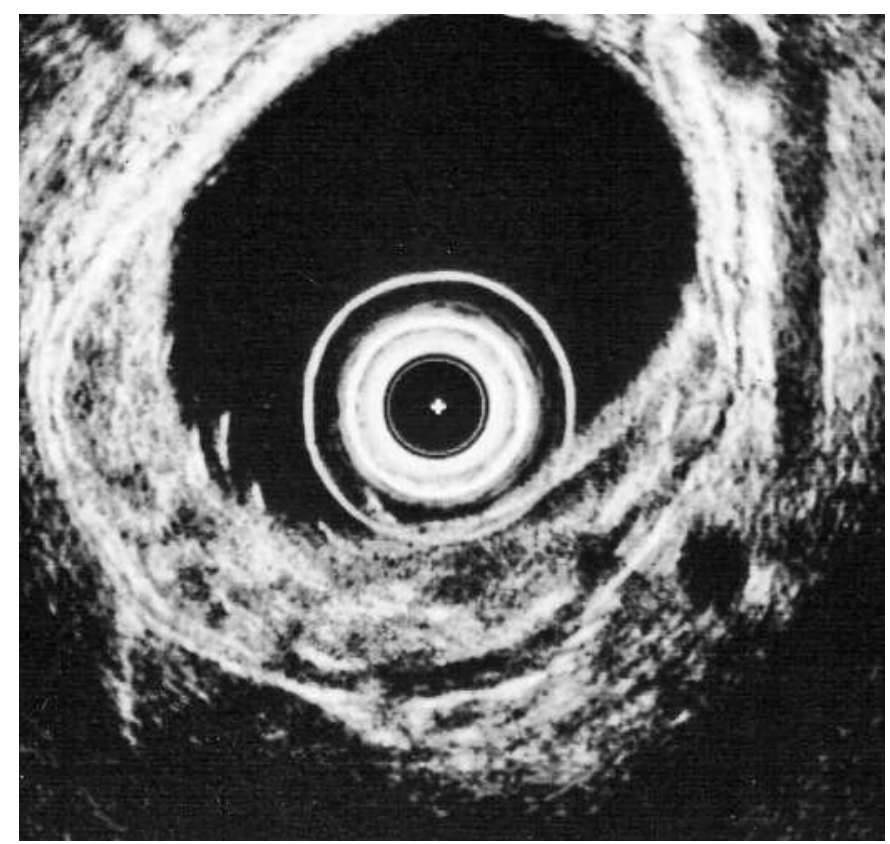

Figure 4 Endoscopic ultrasound view of a giant fold in the fundus, showing multiple anechoic areas in the thickened submucosal layer.

\section{References}

${ }^{1}$ Franzin G, Novelli P. Gastritis cystica profunda. Histopathology 1981; 5: 535-547

${ }^{2}$ Wu MT, Pan HB, Lai PH et al. CT of gastritis cystica polyposa. Abdom Imaging 1994; 19 : 8-10

${ }^{3}$ Okada M, Lizuka Y, Oh K et al. Gastritis cystica profunda presenting as giant gastric mucosal folds: the role of endoscopic ultrasonography and mucosectomy in the diagnostic work-up. Gastrointest Endosc 1994; 40: 640-644

${ }^{4}$ Park JS, Myung SJ, Jung HY et al. Endoscopic treatment of gastritis cystica polyposa found in an unoperated stomach. Gastrointest Endosc 2001; 54: $101-103$

${ }^{5}$ Song YL, Park JY, Kim YB et al. A case of gastritis cystica profunda associated with high grade tubular adenomas. Korean J Gastroenterol 2004; 43: $52-55$
Corresponding author

\section{Béchade, MD}

Department of Gastroenterology

Val de Grâce Hospital

74 Boulevard de Port Royal

75230 Paris

Cedex 05

France

Fax: $\quad$ +33-1-40-51-51-48

Email: bechade.dominique@ wanadoo.fr 